\title{
Relações entre turismo e saúde: abordagens metodológicas e propostas de ação
}

\author{
Vanina Matos ${ }^{1}$ e Christovam Barcellos ${ }^{1}$
}

Como citar Matos V, Barcellos C. Relações entre turismo e saúde: abordagens metodológicas e propostas de ação. Rev Panam Salud Publica. 2010;28(2):128-34.

RESUMO Objetivo. Identificar as relações entre turismo e saúde e as metodologias empregadas em estudos publicados sobre esse tema.

Método. Realizou-se uma busca nas bases PubMed e SciELO em março de 2008 utilizando a seguinte estratégia: traveler or traveller or tourism or tourist AND risk or hazard or vulnerability AND health or surveillance. Foram excluídos os artigos sobre saúde animal, artigos conceituais e de revisão, artigos sobre viajantes que não turistas, artigos escritos em idiomas diferentes de português, inglês, italiano e espanhol. Dos 153 artigos localizados, 112 foram eliminados e 41 foram examinados.

Resultados. O número de artigos sobre o tema cresceu de um artigo na década de 1970 para 34 na década de 2000. A maior parte dos estudos foi realizada na Europa, seguida das Américas, e abordou doenças transmitidas por insetos, doenças transmitidas entre pessoas por via respiratória e doenças gastrointestinais. Foi predominante a utilização de questionários para a coleta de dados, aplicados pessoalmente, por telefone ou por carta. Quanto ao local da pesquisa, 21 estudos foram realizados no local de origem da viagem, 17 no destino e 3 não informaram. Quatro estudos foram realizados antes da viagem, 9 durante a viagem, 24 após a viagem, e 3 não informaram. A maioria dos estudos abordou o turista como vítima preferencial de problemas de saúde, despreparado para enfrentar situações de vulnerabilidade durante a viagem.

Conclusões. É clara a necessidade de implantar políticas de saúde voltadas para o turista, com ênfase em doenças infecciosas e ações emergenciais para detectar surtos envolvendo turistas. Também é necessário um sistema de vigilância e notificação específico para turistas, e maior preparo das instituições de saúde para atender demandas individuais dessa população.

Palavras-chave Saúde do viajante; fatores de risco; política de saúde; vigilância em saúde pública.

A saúde do viajante é uma área que trata dos riscos, individuais e coletivos, ocasionados pela movimentação de pessoas e por sua interação com diversos ambientes. As questões de saúde em viagens vêm sendo, cada vez mais, abordadas por setores de governo, empresas aé-

1 Fundação Oswaldo Cruz, Instituto de Comunicação e Informação Científica e Tecnológica em Saúde (ICICT/FIOCRUZ), Manguinhos, Brasil. Enviar correspondência para Vanina Matos, vanina.matos@gmail.com reas e marítimas e agências de viagem. Entretanto, a maioria dessas ações ainda é restrita a recomendações de vacinação antes da viagem e a descrições sobre riscos veiculadas em páginas da Internet (www.anvisa.gov.br/viajante; www. abav.com.br, www.infraero.gov.br/hora deviajar, www.anac.gov.br/dicasanac).

Segundo as políticas tradicionais de vigilância epidemiológica e sanitária, é justificada a utilização de medidas de prevenção em viajantes, visto que essas medidas podem diminuir o risco de epi- demias. Existem diferentes perfis de viajantes: turistas, profissionais, migrantes, refugiados, soldados e voluntários. Esses viajantes se diferenciam quanto à origem e aos destinos da viagem, objetivos durante a viagem e características individuais. Dessa maneira, o viajante não pode ser encarado como unidade; deve, isso sim, ser reconhecido segundo sua diversidade. Portanto, são necessárias estratégias diferenciadas de atuação para o enfrentamento desse desafio nos sistemas de saúde (1). 
Dentre os diversos perfis de viajantes, é marcante o crescimento do turista. Em todo o mundo, houve um aumento significativo do fluxo turístico entre 1997 e 2007, com mais de 900 milhões de turistas em trânsito, segundo a Organização Mundial de Turismo (OMT). Nesse mesmo período, chegou a 5 milhões o número de desembarques de turistas estrangeiros no Brasil (2). Entre 2001 e 2003 houve uma queda nesse movimento, que pode ser explicada pelos ataques terroristas nos Estados Unidos e pela síndrome respiratória aguda grave, que se espalhou por alguns países da Ásia. Outras emergências de saúde pública podem ter impactado o turismo, como a influenza aviária em 2005 e a influenza suína em 2009. De qualquer modo, o turista adquire, cada vez mais, grande importância nas questões internacionais de vigilância sanitária.

Sendo assim, o objetivo do presente artigo foi revisar a literatura científica para identificar as relações entre turismo e saúde e as metodologias empregadas em estudos sobre esse tema.

\section{MATERIAIS E MÉTODOS}

A busca de bibliografia foi realizada nas bases PubMed (www.pubmed.org) e SciELO (www.scielo.org) em março de 2008. A seguinte estratégia de busca foi utilizada: traveler or traveller or tourism or tourist AND risk or hazard or vulnerability AND health or surveillance. Foram encontrados 153 artigos na PubMed e nenhum na SciELO com essa estratégia de busca.

O termo "traveler" em suas duas grafias (traveler/traveller) foi utilizado além de "tourist", por se tratar de um conceito mais amplo que o de turista. Ainda não existe um padrão conceitual: muitos estudos tratam de turistas sem, no entanto, utilizar o termo tourist. Por outro lado, a movimentação de pessoas não é uma característica específica do turismo, abrangendo também os grupos de migrantes ou outros grupos populacionais como ciganos, exército, voluntários ou refugiados. Foram excluídos os seguintes tipos de artigos: artigos que tratavam de viajantes que não turistas; artigos que tratavam de saúde animal; artigos conceituais e de revisão; artigos escritos em idiomas diferentes de português, inglês, italiano e espanhol.

Após leitura do resumo dos 153 artigos, a aplicação dos critérios de exclusão eliminou 112: em dois o texto na íntegra não foi localizado, 36 eram artigos de revisão e 74 não tratavam especificamente dos turistas ou das atividades de turismo. Assim, 41 artigos foram considerados neste levantamento, um deles traduzido do alemão para o português.

Foram avaliados os seguintes aspectos: tópico do estudo, local de realização (origem ou destino da viagem), ocasião da realização da pesquisa (antes, durante ou depois da viagem), tipologia de turista empregada, metodologia utilizada, problemas de saúde estudados e ações e políticas propostas. Os artigos também foram analisados segundo o ano de publicação e a área da revista em que foram publicados.

\section{RESULTADOS}

Apenas um dos artigos selecionados foi publicado em uma revista da área de turismo (3). Todos os demais foram publicados em revistas da área de saúde (4-43).

Houve um crescimento no número de publicações sobre o tema ao longo dos anos: um artigo na década de 1970; um na década de 1980; cinco na década de 1990; e 34 na década de 2000. O aumento foi marcante entre os anos de 2003 e 2007, com oito artigos publicados em 2004 e 10 em 2005. Em relação à região geográfica dos estudos, 19 foram realizados na Europa, sendo três na França; a Alemanha, a Espanha, a Itália, a Suécia, a Suíça e a Irlanda tiveram dois estudos cada uma; e a Finlândia, a Áustria, a Croácia e a Inglaterra tiveram um artigo. Fora da Europa, África do Sul, Austrália e Cingapura tiveram dois artigos cada. Ainda na Ásia, um estudo foi realizado na China e outro no Nepal, além de um estudo realizado em Israel. Já no continente americano, seis estudos foram realizados nos Estados Unidos, quatro no Brasil, um no México e um na Jamaica. Um estudo não teve relação direta com um determinado país (4) por estar vinculado a um grupo de 15 regiões classificadas dentro de uma Rede de Vigilância Global, estabelecida em 1995 entre membros da International Society of Travel Medicine (ISTM) e dos Centers for Disease Control and Prevention (CDC).

\section{Problemas de saúde}

A classificação de riscos em viagens foi formulada e adaptada de acordo com as categorias propostas no Guia de Vigilância Epidemiológica do Ministério da
Saúde (44) e no Guia de Saúde do Viajante da Petrobras (45). A tabela 1 descreve os problemas abordados. O maior número de estudos abordou as doenças transmitidas por insetos. A malária predominou como tópico em relação a prevenção e conhecimentos do uso de quimioprofilaxia antimalárica antes da viagem para áreas endêmicas e a importação da malária. Um estudo dirigido a diversos problemas de saúde abordou, entre outros, as picadas de insetos como queixa de turistas em decorrência de suas viagens ao estrangeiro (13).

A diarreia do viajante foi classificada como categoria independente em quatro estudos $(8,13,20,21)$, mesmo com transmissão associada à ingestão de água e alimentos contaminados, pois é tratada como problema emergencial pela Organização Mundial da Saúde (OMS) (46) devido ao alto índice de acometimentos, do tempo variado de aparecimento dos sintomas (imediatos ou não) e da dificuldade de identificação do agente patogênico. $\mathrm{Na}$ categoria de alterações psíquicas, acidentes e violência, nenhum estudo tratou de acidentes de trânsito, agressões e assaltos ao turista.

O único estudo que procurou estabelecer relações causais entre exposição e efeito sobre a saúde, relacionando a duração da viagem aérea e a incidência de trombose venosa (37), foi classificado na categoria de "riscos relacionados ao meio de transporte". A categoria "outros" incluiu os riscos não relacionados diretamente com a classificação proposta, como terrorismo, instabilidade política, barreiras culturais, dogmas religiosos, clima, problemas de consumo excessivo de álcool, automedicação e problemas de saúde em geral vinculados a viagens, como o jet lag e o desconforto relacionado à viagem, sendo que alguns artigos agrupavam diversos desses elementos na mesma pesquisa $(3,13,42,43)$.

\section{Abordagens metodológicas}

A menor amostra incluiu 17 turistas que haviam sido contaminados por esquistossomose (35) e a maior amostra envolveu a aplicação de 12499 questionários sobre consumo arriscado de alimento ou bebida e a ocorrência de diarreia durante a viagem, num levantamento realizado entre março de 1997 e fevereiro de 1998 no setor de embarque de passageiros de um aeroporto (21). Esses dois estudos foram feitos no Brasil. 
TABELA 1. Distribuição dos estudos sobre saúde do turista quanto ao problema estudadoa

\begin{tabular}{|c|c|c|}
\hline \multirow[b]{2}{*}{ Categoria } & \multicolumn{2}{|c|}{ Estudos } \\
\hline & Número & Referência \\
\hline Doenças transmitidas por insetos & 13 & \\
\hline Malária & 8 & $4-11$ \\
\hline Leishmaniose & 2 & 7,12 \\
\hline Dengue & 2 & 4,7 \\
\hline Problemas diversos (picadas de insetos) & 1 & 13 \\
\hline Infecções associadas à contaminação por ingestão de água ou alimentos & 11 & \\
\hline Cólera & 2 & 14,15 \\
\hline Febre tifoide & 3 & $4,9,16$ \\
\hline Hepatite A & 1 & 9 \\
\hline Norovírus & 1 & 17 \\
\hline Infecções parasitárias em geral & 1 & 4 \\
\hline Inflamação gastrointestinal & 2 & 8,18 \\
\hline Contaminação por consumo de água & 1 & 19 \\
\hline Doenças transmitidas entre pessoas por via respiratória & 11 & \\
\hline SARS & 2 & 22,23 \\
\hline Tuberculose & 2 & 4,9 \\
\hline Síndromes respiratórias em geral & 2 & 4,13 \\
\hline Vírus influenza & 1 & 4 \\
\hline Varicela & 1 & 9 \\
\hline Doença do legionário & 1 & 24 \\
\hline Outros problemas de saúde (irritação dos olhos e asma) & 2 & 8,25 \\
\hline Doenças sexualmente transmissíveis & 6 & \\
\hline Aids, HIV, sífilis, gonorreia & 3 & $4,26,27$ \\
\hline Hepatite B & 2 & 9,28 \\
\hline Doenças venéreas em geral & 1 & 13 \\
\hline $\begin{array}{l}\text { Conhecimentos, atitudes e práticas relacionados a prevenção de doenças infecciosas, } \\
\text { vacinação, situações de perigo e aconselhamento pré-viagem }\end{array}$ & 5 & $9,38-41$ \\
\hline Diarreia do viajante & 4 & $8,13,20,21$ \\
\hline Alterações psíquicas, acidentes e violência & 4 & \\
\hline Arranhões, abrasão, bolhas, luxação, distensão muscular e queimaduras de sol & 1 & 25 \\
\hline Afogamentos ou acidentes em geral & 2 & 8,29 \\
\hline Experiências de viagem positivas ou negativas vs. aparecimento de doença coronariana & 1 & 30 \\
\hline Riscos ambientais (visitação a áreas de risco ou "doença aguda de montanha") & 3 & $13,25,31$ \\
\hline Infecções associadas a ferimentos e acidentes com animais & 3 & \\
\hline Ataque por animais selvagens a turistas em safári & 1 & 32 \\
\hline Escabiose & 1 & 33 \\
\hline Proteção sorológica contra a raiva em turistas & 1 & 34 \\
\hline Doenças transmitidas através do contato com a água & 2 & \\
\hline Transmissão de esquistossomose em piscinas de hotéis & 2 & 35,36 \\
\hline Riscos relacionados ao meio de transporte & 1 & 37 \\
\hline Outros & 4 & $3,13,42,43$ \\
\hline
\end{tabular}

Um único estudo definiu a amostra com recorte de gênero. Esse estudo investigou o comportamento sexual de risco entre viajantes do sexo masculino nos Estados Unidos. Os dados foram obtidos através de um questionário anônimo preenchido por 247 homens selecionados em bares e negócios orientados para a comunidade local de homossexuais (26). Uma amostra de conveniência foi utilizada em um estudo com 38 viajantes internacionais que procuraram a clínica de medicina de viagem no Nepal durante a viagem e que haviam recebido vacina contra raiva nos últimos 5 anos (34).

Dentre os instrumentos de coleta de dados empíricos, foi predominante a utilização de questionários, tanto fechados quanto abertos, aplicados pessoalmente, por telefone ou por meio de carta $(16,18$, $20,22,23,25,28,39,42,43)$. A entrevista semiestruturada apareceu em menor escala $(22,39)$.

Dados secundários foram utilizados em três estudos. Um analisou os casos de cólera notificados pelos centros nacionais de vigilância em saúde de países industrializados da Europa, América e Ásia (15). Na França, pesquisadores exploraram uma base de dados de notificação compulsória para avaliar a situação epidemiológica e as tendências quanto à cólera importada para o país (14). Um estudo na Itália pesquisou a notificação de casos de leishmaniose cutânea e identificou quatro casos importados: um do Afeganistão, um da Costa Rica, um do México e um caso de turista que viajou entre Tunísia e Marro$\cos (12)$.

Como parte de um estudo envolvendo quatro países, acerca do impacto epidemiológico global sobre a diarreia do viajante, o Brasil avaliou visitantes à Cidade de Fortaleza, no Ceará (21). A avaliação de risco também foi estudada sob o aspecto da prevenção de doenças em viajantes que visitaram amigos e parentes (9).

Dois estudos realizaram inquéritos: um tratou da associação entre saúde psíquica e saúde física, examinando a relação entre ter abertura a experiências turísticas agradáveis como fator psicológico e as taxas de mortalidade por doença cardíaca coronariana, em um contexto internacional (30); o outro foi um inquérito malacológico para identificar focos de infecção de 
esquistossomose em hotéis de lazer em Minas Gerais, Brasil (36).

A aplicação das pesquisas foi atrelada ao local de origem ou destino da viagem e ao período - antes, durante ou depois da viagem. Dos 41 estudos, 21 enfocaram os turistas no local de origem da viagem $(3,6-11,13,15,16,19,23,24,28,30,37$, $38,40-43), 17$ no destino $(5,12,14,17,18$, $20,21,25,26,29,31-36,39)$ e 3 não informaram onde os dados foram coletados $(4,22,27)$; quanto ao período, quatro foram realizados antes da viagem (38, $40-42), 9$ durante a viagem $(17,20,21,25$, $26,31,33,34,39)$ e 24 após a viagem (3-6, 8-16, 19, 23, 24, 28-30, 32, 35-37, 43). Um estudo coletou dados antes e depois da viagem (7), e 3 não informaram o momento da coleta $(18,22,27)$.

De quatro pesquisas antes da viagem, uma foi aplicada em viajantes que procuraram uma clínica médica de doenças infecciosas para aconselhamento e imunização (42). As outras três foram realizadas em saguões de aeroportos no embarque de passageiros para áreas consideradas de risco para aquisição doenças infecciosas (38, 40, 41). Bares, restaurantes, parques, hotéis e aeroportos no destino da viagem foram utilizados por nove pesquisas realizadas durante as viagens $(17,20,21,25,26,31,33$, 34, 39). Um único estudo comparou dados obtidos antes e depois de viagens para dois grupos: pacientes hospitalizados pós-viagem com diagnóstico de doenças infecciosas vs. viajantes saudáveis pré-viagem (7). A tabela 2 lista a nomenclatura utilizada pelos diferentes estudos para designar as pessoas em trânsito.

\section{Ações e políticas propostas}

Em meio às conclusões ou considerações gerais alcançadas, foram ressaltadas as que enquadram o turista como vítima preferencial de problemas de saúde, que necessita de aconselhamento médico pré-viagem e recomendações sobre vacinação, e consideram que essa população não está adequadamente preparada para as diversas situações de vulnerabilidade durante a viagem $(8,15,28$, $34,38,39,41-43$ ).

A vulnerabilidade do turista relacionada à altitude, aos ferimentos e aos acidentes é reconhecida como um fator relevante a ser estudado $(25,29,31)$. Em relação a doenças transmissíveis, alguns estudos avaliam que o turista pode ser um importante subgrupo epidemiológico na transmissão do HIV e sugerem que os casos poderiam ser reduzidos com o uso de preservativos $(26,27)$. Além disso, a importância do uso de quimioprofilaxia antimalárica é enfatizada pelo grande número de pesquisas que estudaram a malária. Assim, alguns artigos ressaltam a falta de políticas específicas de prevenção ou de um sistema de saúde voltado para viajantes em geral, especialmente no que tange à malária $(6$, 10, 11).

Alguns estudos consideram que o melhor local para atingir os turistas com informações de saúde seria a agência de viagens $(38,40)$, enquanto que outros consideram que as companhias aéreas e as autoridades deveriam ser responsáveis pelo aconselhamento aos passageiros para minimizar riscos de saúde relacionados ao meio de transporte (37). A falta de dados específicos a respeito da saúde de turistas é mencionada como causa da dificuldade de relacionar doenças específicas com as atividades de turismo $(12,13,15,16)$.

Algumas conclusões foram observadas quanto aos aspectos de vigilância, informação, comunicação e programas de saúde, como a necessidade de intensificar programas de educação sobre determinada doença (23) ou de promover ações entre as autoridades de saúde e a população local, com educação, saneamento e informação (36). Foram encontradas considerações claras sobre a necessidade de implantar políticas de saúde e medidas de vigilância quando se trata de gastroenterites e diarreia, como a implantação de ações emergenciais que poderiam prontamente detectar surtos relacionados aos turistas $(19,21)$. Na Jamaica, a introdução de programas de prevenção, segundo os autores, conseguiu reduzir em $72 \%$ a taxa de incidência de diarreia em turistas (20). Também foi apontada a necessidade de ações apropriadas de vigilância em saúde e de notificação específica para turistas $(22,24)$.

\section{DISCUSSÃO}

Diversos países da América do Sul, da África e do Oriente Médio não apareceram no levantamento realizado. Por um

TABELA 2. Nome dado ao viajante ou turista na literatura internacional, 1977 a 2007

\begin{tabular}{|c|c|c|}
\hline \multirow[b]{2}{*}{ Nome } & \multicolumn{2}{|c|}{ Estudos } \\
\hline & Número & Referência \\
\hline Turista & 18 & $\begin{array}{c}5,11,13,19,20 \\
24-27,29-33,35 \\
36,38,43\end{array}$ \\
\hline Viajante & 12 & $\begin{array}{c}7,8,15,16,18 \\
21,23,28,37 \\
40,41,42\end{array}$ \\
\hline Viajante e turista & 2 & 10,34 \\
\hline Visitante & 2 & 17,39 \\
\hline Viajante VFR ${ }^{a}$ & 1 & 9 \\
\hline Mochileiro & 1 & 14 \\
\hline VFR $^{\mathrm{a}}$ imigrante, VFR ${ }^{\mathrm{a}}$ viajante, turista viajante & 1 & 4 \\
\hline Viajante como turista, trabalhador em viagem, migrante e militar & 1 & 12 \\
\hline Turismo de massa, exploradores, independentes e população flutuante & 1 & 3 \\
\hline Viajante de curto período, visitante de longo período, imigrante de áreas endêmicas & 1 & 6 \\
\hline Não caracteriza & 1 & 22 \\
\hline Total & 41 & \\
\hline
\end{tabular}

a VFR = Visiting friends and relatives (em visita a amigos ou familiares). 
lado, essa ausência poderia ser explicada pela falta de publicações desses países nas bases bibliográficas escolhidas. Por outro lado, outras razões prováveis seriam a limitação de recursos ou interesse de pesquisa sobre o tema de saúde e turismo, bem como o baixo fluxo de turistas no país, ou até mesmo a falta de registro de problemas de saúde relacionados ao turismo nacional e estrangeiro.

A publicação da maioria dos trabalhos a partir do ano de 2000 mostra que o interesse pelo tema é crescente em pesquisas científicas. No Brasil, a discussão sobre turismo e saúde foi proposta por infectologistas e sanitaristas da medicina de viagem, o que caracteriza um viés com foco na prevenção a partir de consultas clínicas pré-viagem. Essa visão, embora seja válida como iniciativa de pesquisa, é insuficiente para embasar políticas públicas que precisam lidar também com a vigilância e a atenção à saúde e com o tratamento de problemas já instalados.

A Organização Pan-Americana da Saúde (OPAS) prevê que exista interação entre as áreas de saúde e turismo (47). Para a OMS, a prevenção na saúde do turista não poderia ser responsabilidade somente do governo; essa responsabilidade deveria recair também sobre o indivíduo, que precisa buscar informações sobre o local a ser visitado. Portanto, a responsabilidade seria dividida entre o setor saúde, o setor de turismo e o próprio turista (46).

A análise mostrou uma tendência à realização de estudos sobre a resolução de urgências e emergências de saúde, como no caso do estudo na Jamaica, onde houve a participação do governo na criação de um programa de controle imediato para combater a diarreia dos viajantes. Essa ação pode ser compreendida como uma forma de minimizar os prejuízos econômicos decorrentes do impacto negativo para o turismo local, fonte principal de geração de emprego e renda no país. Nesse contexto, o exemplo da pandemia de influenza A (H1N1) levanta diversas questões relacionadas às ações de quarentena e isolamento como controle e vigilância de fronteira no sentido de proteção do território (48), e não do turista.

Entre os quatro artigos destacados no Brasil, dois trataram em sequência do problema de esquistossomose em área de turismo rural: o primeiro a partir de casos notificados e o segundo a partir de investigação no local para confirmar a suspeita. Essa demanda possivelmente foi deflagrada pela vigilância epidemiológica local, que não somente é responsável pela notificação compulsória, mas também tem um papel essencial no fluxo de informações para gerar ação. Nesse sentido, a existência de informações em saúde específicas da população de turistas (nacionais e internacionais) nos sistemas de informação em saúde (como o Sistema de Informação de Agravos de Notificação, o SINAN, no Brasil), possibilitaria conhecer a magnitude dos riscos por destino e por tipo de viagem, dentre outros fatores que facilitariam o direcionamento de ações de vigilância. Esses dados relacionados ao contexto social, econômico e ambiental poderiam estabelecer parâmetros de ordem financeira, de gestão e de organização para subsidiar estratégias na construção de uma política de saúde do turista.

A classificação mais frequente das pessoas em trânsito foi como turistas ou viajantes. Entretanto, percebe-se que não há consenso ou preocupação de conceituar o sujeito que está sendo estudado. São necessários novos estudos teóricos que possam melhorar essa classificação e assim contribuir com explicações mais específicas para a dinâmica do processo de saúde-doença envolvida no deslocamento de pessoas. A geografia da saúde, por exemplo, propõe modelos explicativos dessa dinâmica, baseando-se na ecologia humana da doença, que se preocupa com as formas do comportamento humano no seu contexto cultural e socioeconômico e com a interação com o ambiente para produzir ou prevenir doenças (49).

O predomínio das pesquisas na origem e após a viagem mostra que, em geral, os estudos têm foco na verificação dos fatos ocorridos. Uma prática metodológica predominante foi a aplicação de questionários em aeroportos, clínicas de medicina de viagem e hotéis. Houve uma grande variabilidade de estratégias de amostragem. Não foram encontrados estudos que utilizassem o geoprocessamento, apesar do potencial dos sistemas de informação geográfica para o estudo da saúde do turista (50). O geoprocessamento é um instrumento importante para auxiliar na abordagem geográfica de fluxos e na visualização de dados espaciais.

Apenas um artigo, além de entrevistar turistas, incorporou pessoas das áreas de turismo e de saúde na mesma pesquisa, tendo ouvido guias de turismo, proprietários de hotéis e profissionais de saúde de uma mesma localidade (39). Isso mostra uma lacuna significativa nos estudos existentes quanto ao papel dos gestores do setor turismo na prevenção de riscos à saúde do turista.

A área de saúde tem um papel fundamental no estreitamento das relações entre o turismo e outros setores do governo. Embora seja reconhecida a relação entre as atividades de turismo e suas consequências para a saúde coletiva, a colaboração entre a área de turismo e a de saúde não tem ocorrido historicamente e, em geral, não existe interação entre os órgãos envolvidos $(1,51)$.

\section{CONSIDERAÇÕES FINAIS}

Existem diversos conceitos de risco inerentes a várias disciplinas. Para o senso comum, a conceituação de risco tem uma acepção de ameaça ou perigo, ou mesmo de probabilidade ou incerteza (52). O risco associado à saúde do turista depende de diversos fatores individuais $(53,54)$, sendo que outros fatores coletivos ou globais influenciam a dinâmica do deslocamento de pessoas e a saúde pública. Certamente, os riscos ao turista serão diferentes se comparados aos enfrentados pelo viajante, migrante, refugiado, voluntário e outros que apresentam características distintas de perfil e de tipo de viagem. Os riscos associados ao turismo são extremamente diversificados e dependem da relação entre o turista e o lugar visitado. Os objetivos da viagem e as predisposições do turista são determinantes desses tipos de risco.

Pode-se dizer que a saúde do turista é vista sob dois aspectos, primeiro quanto à preocupação com a saúde individual do turista e segundo sob o ponto de vista de impactos na saúde pública resultantes da intensificação do fluxo de turistas e da interação do turista com o ambiente.

Neste levantamento, observou-se que os estudos abordam o turista frequentemente como uma vítima preferencial de problemas de saúde, pelos seguintes motivos: não possui imunidade para infecções; tem um comportamento que o expõe mais e não domina os códigos locais; o sistema de saúde no local de destino não está preparado para atendê-lo; o sistema de saúde no local de origem não tem políticas específicas de prevenção; o turista não detém as informações neces- 
sárias sobre os riscos a que poderá se expor na viagem. Outros artigos apresentam uma visão do turista como agente de disseminação de doenças, ajudando a difundir agentes infecciosos porque circula de lugares endêmicos para não endêmicos. Poucos artigos consideraram o turista como uma pessoa qualquer, que não possui riscos específi- cos, o que corrobora a necessidade de se implantar uma política específica para esse grupo. É essencial abordar a saúde do turista com um conjunto de ações que deve abranger os governos, as agências de viagem e outras empresas do ramo e também os indivíduos, tanto os próprios turistas quanto aqueles que irão recepcioná-los.
Agradecimentos. Este estudo é parte da tese intitulada "A saúde do viajante na visão de três atores: gestores da saúde pública, gestores do turismo e o turista". O projeto teve apoio da Fundação Oswaldo Cruz na forma de bolsa de doutorado (VM). CB é bolsista de produtividade do Conselho Nacional de Desenvolvimento Científico e Tecnológico (CNPq).

\section{REFERÊNCIAS}

1. Carta de São Paulo. Em defesa da saúde do viajante. 5 set 2008. Disponível em: http:// www.anvisa.gov.br/divulga/noticias / 2008/120908.htm. Acessado em 28 março de 2009.

2. Brasil, Ministério do Turismo, EMBRATUR. Estatísticas básicas de turismo 2008. Brasília, DF: Ministério do Turismo; 2008. Disponível em: braziltour.com/site/arquivos/dados fatos/estatisticas_basicas_do_turismo/esta tiscas basicas do turismo brasil 2003 a 2007__fonte____ 2 set2008_internet.pdf. Acessado em 29 março de 2009.

3. Lepp A, Gibson H. Tourist roles, perceived risk and international tourism. Ann Tourism Res. 2003;30(3):606-24.

4. Leder K, Tong S, Weld L, Kain KC, WilderSmith A, von Sonnenburg F, et al. Illness in travelers visiting friends and relatives: a review of the GeoSentinel Surveillance Network. Clin Infect Dis. 2006;43(9):1185-93.

5. Lehky Hagen MR, Haley TJ, Christoph Hatz FR. Factors influencing the pattern of imported malaria. J Travel Med. 2005;12(2):72-9.

6. Spinazzola F, Nicastri E, Vlassi C, Ghirga P, De Marco M, Pittalis S, et al. Imported malaria at Italy's National Institute for Infectious Disease Lazzaro Spallanzani, 1984-2003. Eur J Clin Microbiol Infect Dis. 2006;26(3):175-9.

7. Stienlauf S, Segal G, Sidi Y, Schwartz E. Epidemiology of travel-related hospitalization. J Travel Med. 2005;12(3):136-41.

8. Rack J, Wichmann O, Kamara B, Günther M, Cramer J, Schönfeld C, et al. Risk and spectrum of diseases in travelers to popular tourist destinations. J Travel Med. 2005;12(5): 248-53.

9. Angell SY, Behrens RH. Risk assessment and disease prevention in travelers visiting friends and relatives. Infect Dis Clin North Am. 2005;19(1):49-65.

10. Raju N, Poljak I, Troselj-Vukic B. Malaria, a travel health problem in the maritime community. J Travel Med. 2000;7(6):309-13.

11. D'Ortenzio E, Bonnet D, Leport C, Le Bras J. Falciparum malaria acquired by a French tourist in a resort area of the Dominican Republic. Euro Surveill. 2005;10(10):E051013.4.

12. Antinori S, Gianelli E, Calattini S, Longhi E, Gramiccia M, Corbellino M. Cutaneous leishmaniasis: in increasing threat for travellers. Clin Microbiol Infect. 2005;11(5):343-6.

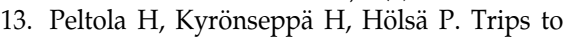
the south-a health hazard. Morbidity of
Finnish travelers. Scand J Infect Dis. 1983; 15(4):375-81.

14. Tarantola A, Ioos S, Rotureau B, Paquet C, Quilici ML, Fournier JM. Retrospective analysis of the cholera cases imported to France from 1973 to 2005. J Travel Med. 2007;14(4): 209-14.

15. Wittlinger $F$, Steffen $R$, Watanabe $H$, Handszuh H. Risk of cholera among Western and Japanese travelers. J Travel Med. 1995; 2(3):154-8.

16. Ekdahl K, de Jong B, Andersson Y. Risk of travel-associated typhoid and paratyphoid fevers in various regions. J Travel Med. 2005; 12(4):197-204.

17. Michel A, Fitzgerald R, Whyte D, Fitzgerald A, Beggan E, O'Connell N, et al. Norovirus outbreak associated with a hotel in the west of Ireland, 2006. Euro Surveill. 2007;12(7):E11-2.

18. McCormick P, Manning D. Chronic inflammatory bowel disease and the "over-clean" environment: rarity in the Irish "traveler" community. Ir Med J. 2001;94(7):203-4.

19. Paez Jiménez A, Pimentel R, Martinez de Aragón MV, Hernandez Pezzi G, Mateo Ontañon S, Martinez Navarro JF. Waterborne outbreak among Spanish tourists in a holiday resort in the Dominican Republic, August 2002. Euro Surveill. 2004;9(3):449.

20. Ashley DV, Walters C, Dockery-Brown C, McNab A, Ashley DE. Interventions to prevent and control food-borne diseases associated with a reduction in traveler's diarrhea in tourists to Jamaica. J Travel Med. 2004;11(6): 364-7.

21. Cavalcanti A, Clemens SA, Von Sonnenburg F, Collard F, De Clercq N, Steffen R, et al. Traveler's diarrhea: epidemiology and impact on visitors to Fortaleza, Brazil. Rev Panam Salud Publica. 2002;11(4):245-52.

22. Teo P, Yeoh BS, Ong SN. SARS in Singapore: surveillance strategies in a globalizing city. Health Policy. 2005;72(3):279-91.

23. Lau J, Yang X, Tsui H, Pang E. SARS related preventive and risk behaviours practised by Hong Kong-mainland China cross border travellers during the outbreak of the SARS epidemic in Hong Kong. J Epidemiol Community Health. 2004;58(12):988-96.

24. Decludt B, Campese C, Lacoste M, Che D, Jarraud S, Etienne J. Clusters of travel associated legionnaires' disease in France, September 2001-August 2003. Euro Surveill. 2004; 9(2):12-3.
25. Heggie TW, Heggie TM. Viewing lava safely: an epidemiology of hiker injury and illness in Hawaii Volcanoes National Park. Wilderness Environ Med. 2004;15(2):77-81.

26. Benotsch EG, Mikytuck JJ, Ragsdale K, Pinkerton SD. Sexual risk and HIV acquisition among men who have sex with men travelers to Key West, Florida: a mathematical modeling analysis. AIDS Patient Care STDS. 2006;20(8):549-56.

27. Mazzaro JM. Sexual tourism: implications for travelers and the destination culture. Infect Dis Clin North Am. 2005;19(1):103-20.

28. Streeton CL, Zwar N. Risk of exposure to hepatitis B and other blood-borne viruses among Australians who travel abroad. J Travel Med. 2006;13(6):345-50.

29. Bauer R, Körmer C, Sector M. Scope and patterns of tourist injuries in the European Union. Int J Inj Contr Saf Promot. 2005;12(1): 57-61.

30. Gidron Y. The relationship between tourists' agreeableness and openness to experience with coronary heart disease mortality. J Psychosom Res. 2004;57(3):227-9.

31. Honigman B, Theis MK, Koziol-McLain J, Roach R, Yip R, Houston C, et al. Acute mountain sickness in a general tourist population at moderate altitudes. Ann Intern Med. 1993;118(8):587-92.

32. Durrheim DN, Leggat PA. Risk to tourists posed by wild mammals in South Africa. J Travel Med. 1999;6(3):172-9.

33. Fernandez G, Morales E, Beutelspacher $C$, Villanueva A, Ruiz C, Stetler HC. Epidemic dermatitis due to contact with a moth in Cozumel, Mexico. Am J Trop Med Hyg. 1992; 46(5):560-3.

34. Ranney M, Partridge R, Jay GD, Rozzoli DE, Pandey P. Rabies antibody seroprotection rates among travelers in Nepal: rabies seroprotection in travelers. J Travel Med. 2006; 13(6):329-33.

35. Enk MJ, Amorim A, Schall VT. Acute schistosomiasis outbreak in the metropolitan area of Belo Horizonte, Minas Gerais: alert about the risk of unnoticed transmission increased by growing rural tourism. Mem Inst Oswaldo Cruz. 2003;98(6):745-50.

36. Enk MJ, Caldeira RL, Carvalho OS, Schall VT. Rural tourism as risk factor for the transmission of schistosomiasis in Minas Gerais, Brazil. Mem Inst Oswaldo Cruz. 2004;5 Suppl 1:S105-8. 
37. Kelman CW, Kortt MA, Becker NG, Li Z, Mathews JD, Guest CS, et al. Deep vein thrombosis and air travel: record linkage study. BMJ. 2003;327(7423):1072-5.

38. Lopez Velez R, Bayas JM. Spanish travelers to high-risk areas in the tropics: airport survey of travel health knowledge, attitudes, and practices in vaccination and malaria prevention. J Travel Med. 2007;14(5):297-305.

39. Ariza L, Gomide M, Ramos AN Jr., Leggat PA, Heukelbach J. Survey of visitors to a National Park in the savannah region of northeast Brazil: practices, incidents and hazardous situations. Travel Med Infect Dis. 2007;5(1):1-6.

40. Toovey S, Jamieson A, Holloway M. Travelers' knowledge, attitudes and practices on the prevention of infectious diseases: results from a study at Johannesburg International Airport. J Travel Med. 2004;11(1):16-22.

41. Wilder-Smith A, Khairullah NS, Song JH, Chen CY, Torresi J. Travel health knowledge, attitudes and practices among Australasian travelers. J Travel Med. 2004;11(1):9-15.

42. Ahim C, Lundberg S, Fessé K, Wiström J. Health problems and self-medication among
Swedish travellers. Scand J Infect Dis. 1994; 26(6):711-7.

43. Van der Linde F, Steffen R, Schwarzenbach FH. Risikogruppen bei Tropenreisen im Rahmen des Massentourismus. Soz Praventiv Med. 1977;22(4):159-60.

44. Brasil, Ministério da Saúde, Secretaria de Vigilância em Saúde. Guia de vigilância epidemiológica. $6^{\mathrm{a}}$ ed. Brasília: Ministério da Saúde; 2005. Disponível em: bvsms.saude.gov.br/ bvs/publicacoes/Guia_Vig_Epid_novo2.pdf. Acessado em março de 2010.

45. Brasil, Petrobras. Guia de saúde do viajante: para profissionais de saúde da Petrobras. Rio de Janeiro: Petrobras; 2004

46. World Health Organization. International travel and health 2009. Genebra: WHO; 1991. Disponível em: www.who.int/ith. Acessado em 20 fevereiro de 2009.

47. Organización Panamericana de la Salud. Salud y turismo. Washington: Organización Panamericana de la Salud; 1992.

48. Silva LJ. Em defesa do território: quarentena e isolamento como medidas de proteção contra a introdução de doenças transmissíveis. Em: Miranda AC, Barcellos C, Monken M,
Moreira JC. Território, ambiente e saúde. Rio de Janeiro: Fiocruz; 2008. Pp. 77-88.

49. Meade MS, Earickson RJ. Medical geography. $2^{\mathrm{a}}$ ed. Nova Iorque: Guilford; 2000.

50. Bauer IL, Poutinen M. Geographic information systems and travel health. J Travel Med. 2002;9(6):308-14.

51. Dias HP. Direitos e obrigações em saúde. Brasília: Agência Nacional de Vigilância Sanitária; 2002.

52. Castiel LD. On the "concreteness" of risk: from epidemiometric epistemological realism to riskological ontology-to keep the ball rolling. Cad Saude Publica. 2001;17(6):1548-9.

53. Spira AM. Preparing the traveller. Lancet. 2003;361(9366):1368-81.

54. Ryan ET, Kain KC. Health advice and immunizations for travelers. N Engl J Med. 2000; 342(23):1716-25.

Manuscrito recebido em 12 de novembro de 2009. Aceito em versão revisada em 25 de janeiro de 2010.

ABSTRACT Objective. To identify relationships between tourism and health as well as the methods employed in studies about this topic.

Method. The PubMed and SciELO databases were searched in March of 2008 using

Tourism and health: methodological approaches and courses of action vulnerability AND health or surveillance. The following were excluded: articles on animal health, conceptual and review articles, articles about non-tourist travel, and articles written in languages other than Portuguese, English, Italian, and Spanish. Of 153 articles identified, 112 were excluded, and 41 articles were examined.

Results. The number of articles on tourism and health increased from one in the 1970 s to 34 in the 2000s. Most studies were carried out in Europe, followed by the Americas, and most covered insect-borne diseases, respiratory diseases transmitted from person to person, and gastrointestinal diseases. Mail, telephone, or face-to-face questionnaires were generally used for data collection. In terms of location, 21 studies were performed at the place of departure, 17 at the destination, and in 3 this information was not specified. Four studies were carried out before the trip, 9 during the trip, 24 after the trip, and 3 did not specify this information. Most studies focus on the tourist as a likely victim of health problems, unprepared to face situations of exposure during the trip.

Conclusions. The need to implement health care policies aimed at the tourist population is evident, with emphasis on infectious diseases and emergency actions to detect outbreaks involving tourists. A tourist-specific surveillance and notification system is also necessary, together with measures to prepare health care institutions to meet the individual demands of this population.

Key words Travelers' health; health policy; public health surveillance. 